\title{
The Impact of Acquired EGFR T790M Mutation and EGFR Circulating Cell-Free DNA on Survival in Patients with Lung Adenocarcinoma Following EGFR-TKI Therapy
}

This article was published in the following Dove Press journal: OncoTargets and Therapy

\author{
Wen-Chien Cheng ${ }^{\prime}$ \\ Te-Chun Hsia $\mathbb{D}^{1,2}$ \\ Chih-Yen Tu' ${ }^{1,2}$ \\ Hung-Jen Chen (iD) ${ }^{1,2}$ \\ 'Division of Pulmonary and Critical Care, \\ Department of Internal Medicine, China \\ Medical University Hospital, Taichung, \\ Taiwan; ${ }^{2}$ School of Medicine, College of \\ Medicine, China Medical University, \\ Taichung, Taiwan
}

\begin{abstract}
Objective: To utilize liquid biopsy to investigate the potential clinical factors influencing the incidence of the acquired epidermal growth factor receptor (EGFR) T790M mutation, and the impact of $E G F R$ circulating cell-free DNA (CfDNA) on overall survival for patients with advanced $E G F R$-mutated adenocarcinoma resistant to first-line EGFR-tyrosine kinase inhibitor (TKI).
\end{abstract}

Methods: A retrospective study was conducted to analyze EGFR-mutated stage IIIB-IV adenocarcinoma patients who received an EGFR-TKI (gefitinib, erlotinib, or afatinib) as first-line therapy and then underwent a liquid biopsy exam at disease progression.

Results: A total of 135 patients were included, and the T790M mutation was detected in 51 patients $(37.7 \%)$. The incidence of T790M mutation increased with the number of initial metastatic sites $(p=0.015)$. Liver metastasis (odds ratio [OR], 3.373; $p=0.017$ ) and other metastasis $(\mathrm{OR}, 3.063 ; p=0.023)$ were also independently correlated with T790M mutation incidence. T790M mutation was also associated with more than two progressive sites (OR, 3.382; $p=0.006$ ), liver progression (OR, 6.204; $p=0.002$ ), and bone progression (OR, $3.366 ; p=0.004)$. However, central nervous system progression was inversely correlated with T790M mutation (OR, 0.183; $p=0.027$ ). Overall survival was the longest among the patients without CfDNA, followed by those shedding T790M mutation and those shedding Del 19/L858R mutations ( $p=0.005)$.

Conclusion: Initial metastasis to the liver and other sites may be independent factors for secondary EGFR T790M mutation. T790M-positive lung adenocarcinoma has specific progression patterns. Moreover, not having EGFR CfDNA, being positive for Del19/L858R mutations, and being positive for T790M mutation have differing impacts on overall survival for patients with advanced $E G F R$-mutated adenocarcinoma resistant to first-line EGFR-TKI. Keywords: circulating cell-free DNA, EGFR, liquid biopsy, overall survival, T790M

\section{Introduction}

In the last decade, non-small-cell lung cancer (NSCLC), especially adenocarcinoma, has been recognized as comprising a heterogeneous group of malignancies with different molecular patterns. ${ }^{1}$ In patients with adenocarcinoma whose tumors harbor epidermal growth factor receptor (EGFR) mutations, first-generation EGFRtyrosine kinase inhibitors (EGFR-TKIs, ie, gefitinib and erlotinib) ${ }^{2,3}$ and secondgeneration EGFR-TKI (such as afatinib) ${ }^{4,5}$ have offered therapeutic options for
Correspondence: Hung-Jen Chen Division of Pulmonary and Critical Care, Department of Internal Medicine, China Medical University Hospital, No. 2, Yude Road, North District, Taichung City 40447, Taiwan

Email redman0127@gmail.com
OncoTargets and Therapy 2020:13 13425-13435

13425

submit your manuscript

DovePress if in

http://doi.org/10.2147/0TT.S279540 
EGFR-mutated patients. However, most cancers still progress after a median response period of 10-12 months, mainly due to T790M mutation. ${ }^{6}$ Osimertinib, a thirdgeneration EGFR-TKI, has shown promising results in treating EGFR T790M-mutated lung cancer, with a median progression-free survival (PFS) of 10.1 months. $^{7}$

Nevertheless, only around $50 \%$ of cases show acquired resistance to first- and second-generation EGFR-TKIs via T790M mutation. ${ }^{6}$ Osimertinib is also used as a first-line therapy in lung cancer patients harboring EGFR mutations and has been shown to be beneficial in terms of delaying the development of resistance, with a median PFS of 18.9 months. ${ }^{8}$ However, the determination of a suitable standard therapy after acquired resistance to osimertinib remains a challenge. ${ }^{9}$

A previous study found that first-line treatment with ramucirumab plus erlotinib in patients with EGFR-mutated advanced NSCLC resulted in a median PFS of 19.4 months. The proportion of patients with T790M mutation at progression who received ramucirumab plus erlotinib was similar to the proportion of patients who received erlotinib alone, and in such cases, treatment with osimertinib continues to be an option. ${ }^{10}$ Therefore, it is crucial to identify potential clinical factors associated with developing T790M mutation. In this group of patients with the potential for developing T790M mutation, a firstor second-generation EGFR-TKI with antiangiogenic agents followed by osimertinib may be a better treatment option.

Re-biopsy to detect the T790M mutation at progressive disease (PD) following first-line EGFR-TKI treatment is essential to guide clinical decisions, although identifying the T790M mutation may be challenging in clinical practice. ${ }^{11}$ Previously reported data from tissue re-biopsy studies indicated that EGFR 19 deletion (Del 19), ${ }^{12}$ longer PFS with first-line EGFR-TKI, ${ }^{13,14}$ and the use of gefitinib/erlotinib ${ }^{14,15}$ might predict the development of T790M-positive disease.

However, these results might have potential selection bias because progressive sites may not be amenable to biopsy (eg, CNS sites), and coexisting medical conditions may preclude biopsies. Furthermore, a single tissue rebiopsy cannot cover the entirety of a heterogeneous tumor. ${ }^{16}$ In contrast, a liquid biopsy to detect circulating cell-free DNA (CfDNA) EGFR genotyping using plasma specimens may overcome the inadequacy of sample quality and tumor heterogeneity associated with tissue biopsies. Furthermore, the cobas plasma test that detects $E G F R$ mutations has demonstrated acceptable agreement between plasma and tissue biopsies. ${ }^{17}$

To date, most studies have used tissue rebiopsies $^{13,15,18}$ or mixed tissue and liquid biopsies ${ }^{14,19,20}$ to investigate the clinical factors influencing the incidence of T790M mutation. Only a few studies so far, however, have used liquid biopsies only to investigate this problem. $^{21,22}$ Furthermore, Taus et al reported that the PFS of lung cancer patients treated with EGFR-TKIs in whom EGFR CfDNA was not detected in plasma was significantly longer than that of those in whom EGFR CfDNA remained detectable during treatment, which meant that the EGFR CfDNA dynamics were positively correlated with radiologic progression. ${ }^{22}$ However, none of those previous studies explored the survival impact from the viewpoint of liquid biopsy results (that is, in patients shedding Del19/L858R mutations, shedding T790M mutations, or in whom EGFR CfDNA was not detected) in patients who experienced PD following first-line EGFRTKI treatment. Therefore, the aim of the current study was to utilize liquid biopsy, a relatively objective diagnostic method, to investigate potential clinical factors influencing the incidence of T790M mutation and to determine the impact of EGFR mutant CfDNA results on the overall survival of patients with advanced EGFR-mutated adenocarcinoma resistant to first-line EGFR-TKI.

\section{Materials and Methods Study Participants}

We performed a retrospective study to analyze EGFRmutated stage IIIB-IV adenocarcinoma patients who received an EGFR-TKI (gefitinib, erlotinib, or afatinib) as first-line therapy and subsequently underwent a circulating cfDNA exam at PD between December 2014 and March 2020 at China Medical University Hospital. Patients who received firstand second-generation EGFR-TKIs, took osimertinib before undergoing a liquid biopsy, or who had a de novo T790M mutation were excluded. The Institutional Review Board of China Medical University Hospital approved this study (CMUH 109-REC-054), and informed consent was waived due to the observational and retrospective design.

\section{Clinical Data Collection, Clinical Assessments, and Efficacy Evaluations}

We assessed clinical factors by classification into the following three categories: (1) Demographic and clinical data, 
including age, sex, and smoking history, were collected. Patients who had never smoked or who had smoked $<100$ cigarettes in their lifetime were categorized as non-smokers. (2) Lung cancer-related information, including the histological type, stage (8th edition of the Classification of Malignant Tumors), ${ }^{23}$ Eastern Cooperative Oncology Group performance status (ECOG-PS), ${ }^{24}$ type of sensitizing EGFR mutation, baseline metastatic sites, EGFR-TKI treatment, PFS during TKI therapy, post-PD chemotherapy history, and timing of liquid biopsy, was also recorded. (3) The progressive pattern and overall survival results were likewise collected. Other metastasis was defined as metastases not including lymph node metastasis, lung-to-lung metastasis, liver metastasis, adrenal gland metastasis, bone metastasis, pleura metastasis, or central nervous system (CNS) metastasis. PFS was measured as the period from the initiation date of EGFR-TKI treatment to the date of radiologic or clinical evidence of progression or death. Overall survival was defined as the time from lung cancer diagnosis to death of any cause.

\section{EGFR Mutation with CfDNA}

At $\mathrm{PD}$, ten $\mathrm{mL}$ of blood was collected in CfDNA collection tubes and centrifuged for 20 minutes at $3000 \mathrm{rpm}$ at room temperature within 36 hours of the blood sample being taken. Plasma samples were processed, and circulating CfDNA was isolated using the cobas ${ }^{\circledR}$ CfDNA sample preparation kit. The target DNA was then amplified and detected on a cobas z 480 analyzer using the amplification and detection reagents provided in the cobas ${ }^{\circledR}$ EGFR Mutation Test v2 kit (Roche). Full details of the method have been reported previously. ${ }^{25}$ Some patients were immediately examined by liquid biopsy after a failed first-line EGFR-TKI treatment, while the others were examined by liquid biopsy after second-line treatment with chemotherapy. The former were defined as having had an immediate liquid biopsy.

We classified the liquid biopsy results into three categories as follows: group A: the shedding Del 19/L858R group, ie, the patients who showed persistence of the original EGFR mutation; group B: the undetected group, ie, the patients had no detectable $E G F R$-activating mutation or T790M mutation in plasma; and group C: the shedding T790M group, ie, the patients in whom a T790M mutation was newly detected.

\section{Statistical Analyses}

For clinical data descriptions, continuous variables were reported as means with standard deviations or medians with interquartile ranges (IQR; 25th and 75th percentiles), and categorical variables were expressed as percentages. The chi-square test was used to compare the differences between the independent groups. Multivariate logistic regression analysis was used to evaluate the factors further independently associated with T790M mutation incidence. A receiver operating characteristic (ROC) curve analysis was performed to transform continuous variables into categorical variables. The overall survival was estimated using the Kaplan-Meier method, and differences among the subgroups of liquid biopsy results were compared using the Log rank test. A $p$-value $<0.05$ was set as statistically significant. All statistical analyses were analyzed using MedCalc for Windows version 18.10 (MedCalc Software, Ostend, Belgium).

\section{Results}

A total of 147 patients who started treatment with EGFRTKIs before PD during the study period were identified. After excluding seven patients treated with two EGFRTKIs and five patients who took osimertinib before undergoing a liquid biopsy, one hundred and thirty-five patients were finally included in the study sample. The clinical characteristics of those patients are summarized in Table 1. The patients' median age was 63.2 years, and they included 87 females $(87 / 135,64.4 \%)$ and 101 never smokers $(101 / 135,74.8 \%)$. A total of $40(40 / 135,29.6 \%)$ patients received gefitinib, $50(50 / 135,37.0 \%)$ received erlotinib, and $45(45 / 135,33.3 \%)$ received afatinib. Thirtysix $(36 / 135,26.7 \%)$ patients were treated with chemotherapy before undergoing a liquid biopsy, and 99 (99/135, $73.3 \%$ ) received an immediate liquid biopsy at PD. A total of $31(31 / 135,23 \%)$ patients showed persistence of the original EGFR mutation (the shedding Del 19/L858R group), and the T790M mutation was newly detected in 51 patients $(51 / 135,37.7 \%)$ (the shedding T790M group). Fifty-three $(53 / 135,39.3 \%)$ patients had no detectable EGFR-activating mutation or the T790M mutation in plasma (the undetected group).

The likelihood of T790M detection was lower in the subcategory of patients with stage IVa disease (10/46, $21.7 \%)$ than in those with IVb disease $(38 / 85,44.7 \%)$. As shown in Figure 1, the incidence of T790M mutation increased consistently with the initial number of metastatic sites present ( $p=0.015$ ) (Figure 1).

As shown in Table 2, the T790M-negative group was regarded as group A (shedding Del 19/L858R) plus group 
Table I Patient Baseline Clinical Factors

\begin{tabular}{|c|c|}
\hline Characteristics & Values \\
\hline Age (year) mean \pm SD & $63.2 \pm 11.4$ \\
\hline Sex (male), n (\%) & $48(35.6)$ \\
\hline \multicolumn{2}{|l|}{ Smoking, n (\%) } \\
\hline Current & $14(10.4)$ \\
\hline Former & $15(11.1)$ \\
\hline Never & I0I (74.8) \\
\hline Unknown & $5(3.7)$ \\
\hline \multicolumn{2}{|l|}{ Histology, n (\%) } \\
\hline Adenocarcinoma & I33 (98.5) \\
\hline Others* & $2(1.5)$ \\
\hline \multicolumn{2}{|l|}{ Stage, n (\%) } \\
\hline IIlb & $4(3)$ \\
\hline $\mathrm{IVa}$ & $46(34.1)$ \\
\hline $\mathrm{IVb}$ & $85(63)$ \\
\hline \multicolumn{2}{|l|}{ EGFR Mutation, n (\%) } \\
\hline Del 19 & $73(54.0)$ \\
\hline L858R & $58(43.0)$ \\
\hline Uncommon $^{+}$ & $4(3.0)$ \\
\hline \multicolumn{2}{|l|}{ ECOG, n (\%) } \\
\hline $0-1$ & $123(9 \mid .1)$ \\
\hline$\geq 2$ & $6(4.4)$ \\
\hline Unknown & $6(4.4)$ \\
\hline \multicolumn{2}{|l|}{ First line TKI treatment, $\mathrm{n}(\%)$} \\
\hline Gefitinib & $40(29.6)$ \\
\hline Erlotinib & $50(37.0)$ \\
\hline Afatinib & $45(33.3)$ \\
\hline Chemotherapy, n (\%) & $36(26.7)$ \\
\hline \multicolumn{2}{|l|}{ Liquid Biopsy, n (\%) } \\
\hline T790M & $2(1.5)$ \\
\hline T790M + Del I9/L858R & $49(36.3)$ \\
\hline Del I9/L858R & $31(23)$ \\
\hline Unfound & $53(39.3)$ \\
\hline
\end{tabular}

Notes: *Others: I Spindle cell carcinoma mixed adenocarcinoma, I adenosquamous carcinoma. 'Uncommon mutation: I L86IQ and G724S, I Del 19 and G7I9S, I G719X, I L747P

Abbreviations: EGFR, epidermal growth factor receptor; TKI, tyrosine kinase Inhibitors; ECOG, Eastern Cooperative Oncology Group; SD, standard deviation.

B (undetected), and the T790M-positive group was regarded as group $\mathrm{C}$ (shedding T790M). The clinical factors of age, smoking, sex, PFS, and receiving an immediate liquid biopsy were not associated with the detection of T790M after acquired resistance to EGFR-TKI. A total of 27 patients with a Del 19 mutation $(27 / 73,37.0 \%)$ and 24 patients with an L858R mutation (24/57, 41.4\%) acquired a T790M mutation $(p=0.608)$. T790M mutation was not detected in any of the three patients with uncommon

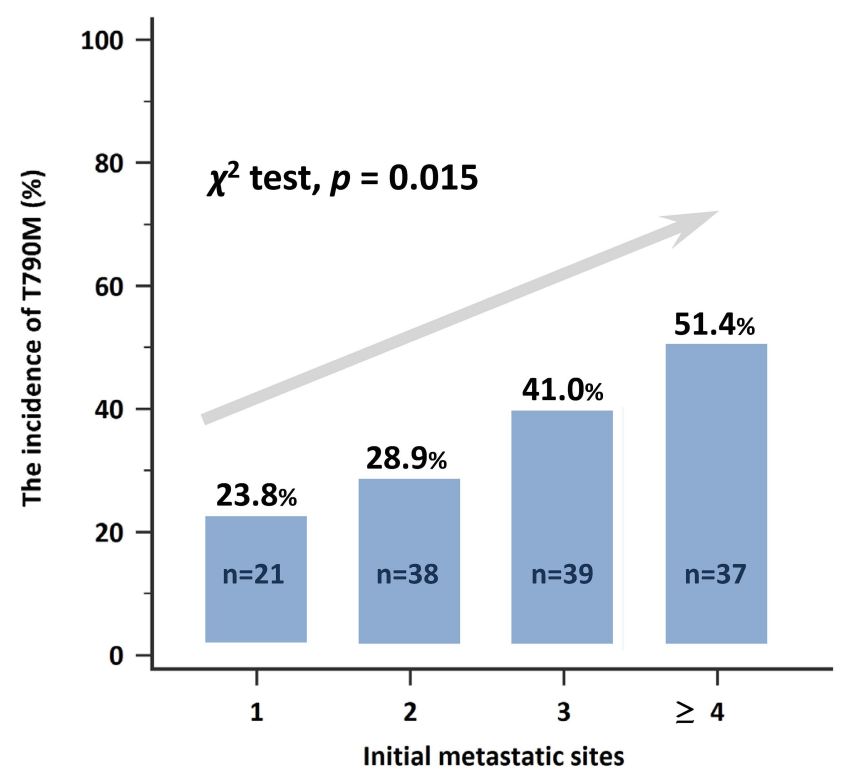

Figure I T790M-positivity directly increases with the initial number of metastatic sites present.

$E G F R$ mutations. We found a slightly higher incidence of the T790M mutation in patients treated with gefitinib or erlotinib than in those treated with afatinib (36 [40\%] versus $15[33 \%]$ subjects, respectively), although the difference did not reach statistical significance $(p=0.449)$.

Before the logistic regression analysis, we constructed a ROC curve for age and PFS. The optimal cut-off value for age was 70 years, with a sensitivity of $29.4 \%$ and specificity of $79.8 \%$. The optimal cut-off value for PFS was 13 months, with a sensitivity of $74.5 \%$ and specificity of $36.9 \%$. Thus, PFS $<13$ months and age $>70$ were selected as categorical variables in the follow-up analysis. In the univariate analysis, T790M mutation detection was correlated with the following factors: liver metastasis ( $p=$ $0.011)$, bone metastasis $(p=0.019)$, and other metastasis (that is, pericardial effusion and chest wall, spleen, pancreas, kidney, peritoneum, and soft tissue metastasis) ( $p=$ 0.012). In the multivariate analyses, clinically important variables from previous reports (age, PFS, EGFR mutation, EGFR-TKIs), ${ }^{12-15,19,20}$ as well as variables with $\mathrm{p}<$ 0.20 in the univariate analysis (liver metastasis, bone metastasis, other metastasis, and immediate liquid biopsy), were included in the final model. Liver metastasis (odds ratio [OR], 3.373; 95\% confidence interval [CI], 1.244 to 9.143; $p=0.017$ ) and other metastasis (OR, 3.063; 95\% CI, 1.111 to $8.086 ; p=0.023$ ) had significant independent correlations with the incidence of $\mathrm{T} 790 \mathrm{M}$ mutation (Figure 2). 


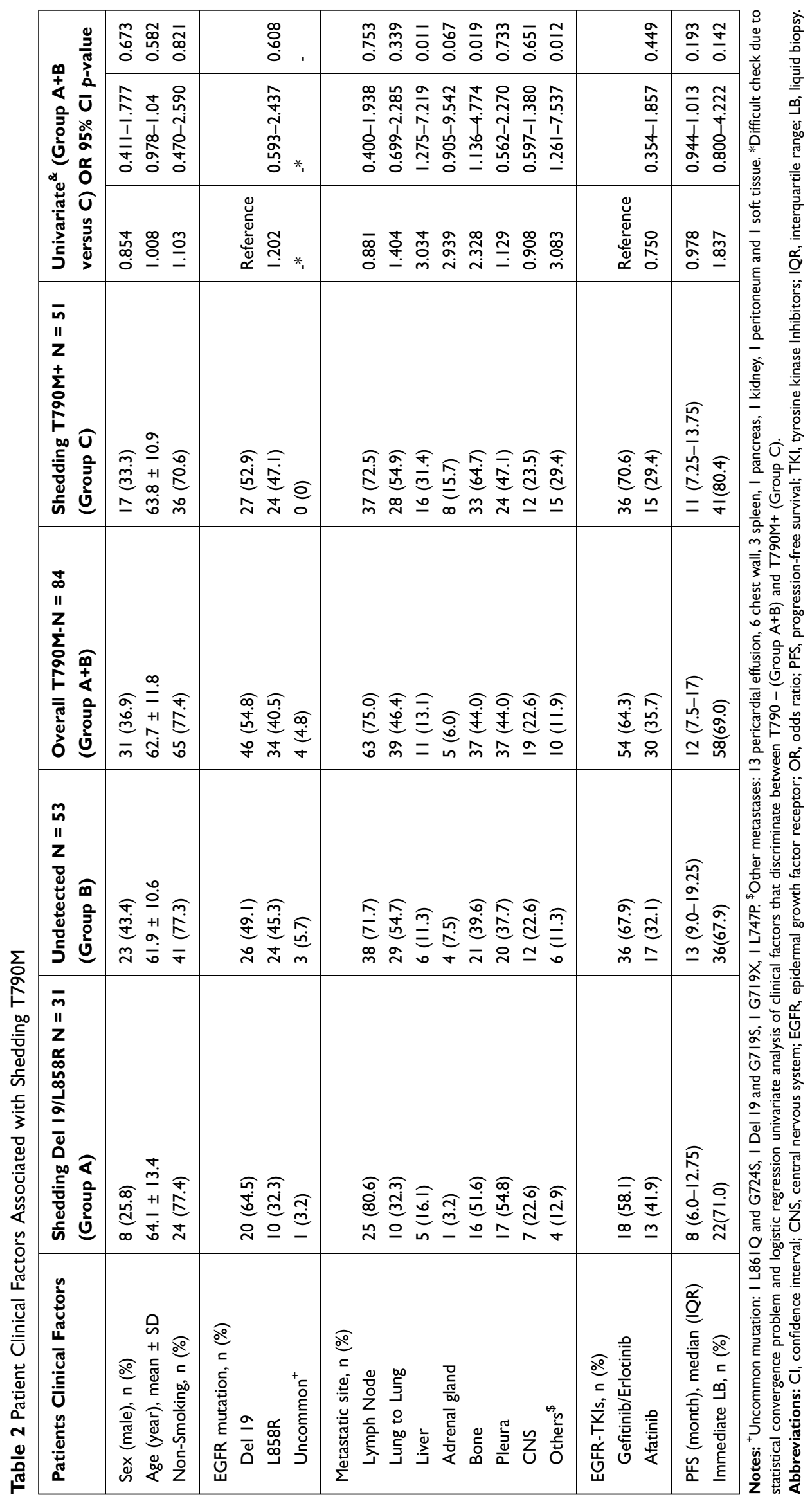




\section{T790M analysis}

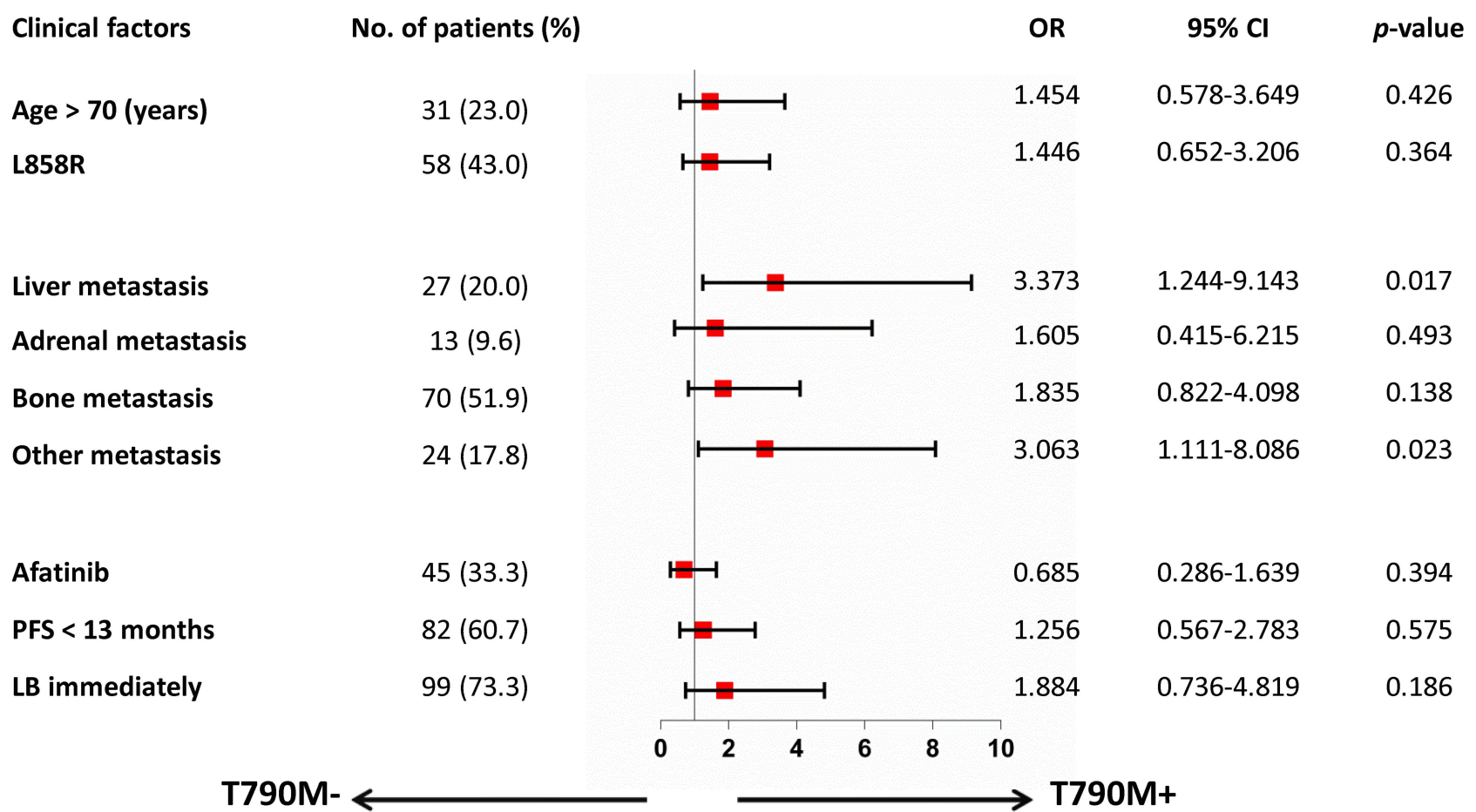

Figure 2 Forest plot for the T790M mutation subgroup (multivariate analysis).

Abbreviations: LB, liquid biopsy; PFS, progression-free survival.

Regarding the progression pattern with the acquired T790M mutation, as shown in Figure 3, T790M mutation was associated with two or more progressive sites (OR, 3.841; $p=0.007)$, liver progression (OR, 9.297; $p=$ 0.003 ), and bone progression (OR, 3.530; $p=0.010)$. In contrast, CNS progression was inversely correlated with T790M mutation (OR, $0.183 ; p=0.027$ ).

Overall survival was found to be the longest among the undetected group (median, 115.6 months; 95\% CI, not reached), followed by the shedding T790M group (median, 34.2 months; 30.3-34.2), and the shedding Del 19/ L858R group (23.9 months; 20.9-42.9; $p=0.005$ ) (Figure $4 \mathrm{~A})$. We further explored the survival outcomes stratified by those accepting osimertinib treatment after PD under first-line EGFR-TKI treatment. A total of 78 (78/135, 57.8\%) patients accepted osimertinib treatment. Among those patients, there were 35 CfDNA T790M-negative patients who accepted osimertinib treatment, twelve of whom received tissue re-biopsies. One of those patients was found to be T790M-positive, and the others were persistent in being T790M-negative. The undetected and shedding T790M group patients who accepted osimertinib treatment had longer overall survival durations (54.6 months and not reached, respectively) than the shedding Del 19/L858R group (22.9 months), although the differences were not statistically significant (Figure 4B). Fiftyseven $(57 / 135,42.2 \%)$ patients did not accept osimertinib treatment at $\mathrm{PD}$, and among those patients, no overall survival difference was observed between the shedding Del 19/L858R and T790M groups (24.9 months versus 29.1 months; $p=0.853$ ). However, the undetected group's overall survival was significantly longer than that of the Del 19/L858R group (median not reached versus 24.9 months, $p=0.008$ ) (Figure 4C).

\section{Discussion}

The present study was a relatively large cohort real-world study that focused on liquid biopsy and the survival impact of acquired EGFR T790M mutation and EGFR CfDNA after first-line EGFR-TKI treatment on patients with advanced adenocarcinoma with disease progression. The T790M mutation was detected in $37.7 \%$ of the patients, and the incidence of the mutation increased consistently with the initial number of metastatic sites $(p=0.015)$. 


\section{T790M with progressive pattern}

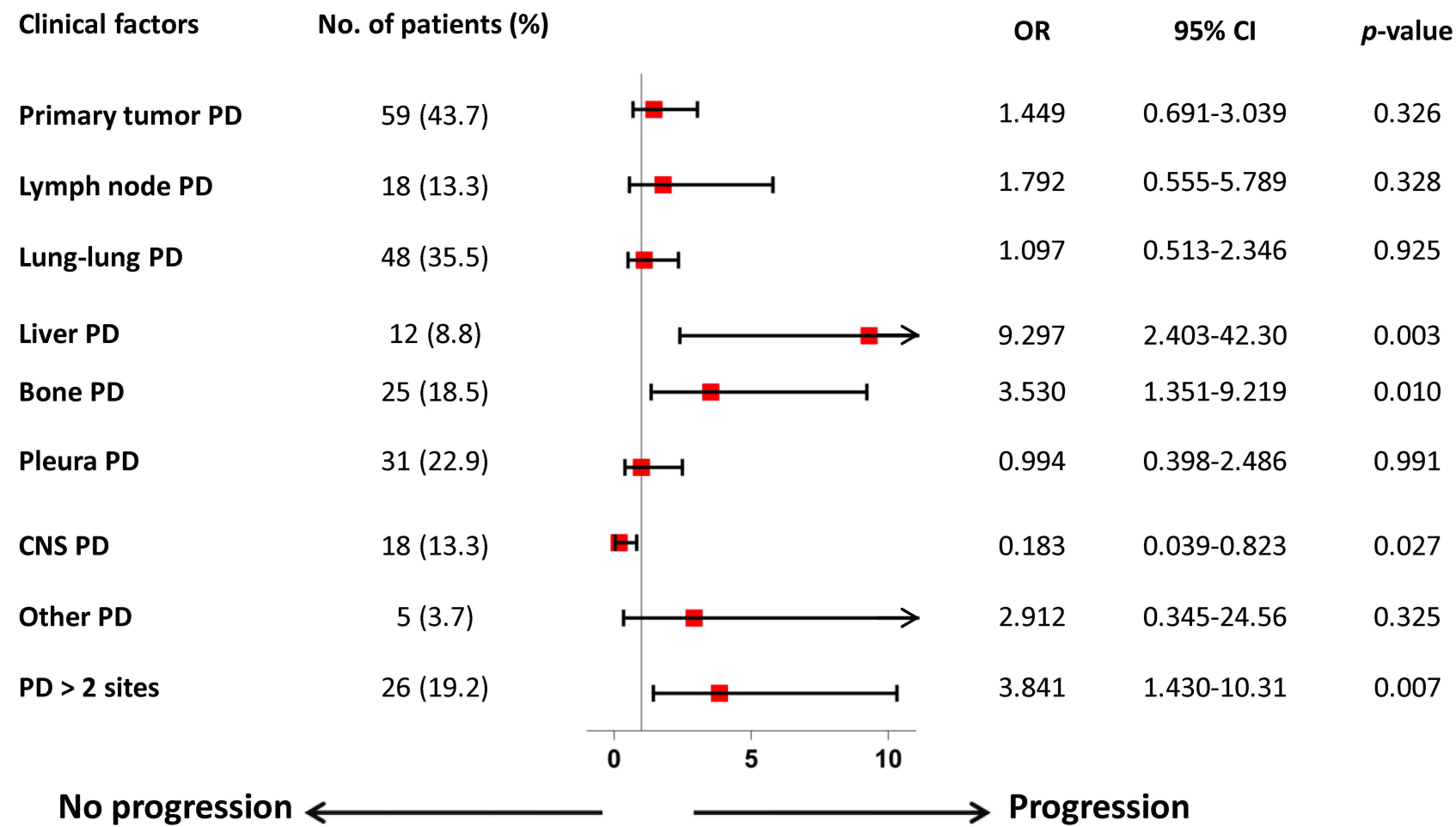

Figure 3 Forest plot for T790M mutation with a progressive pattern (multivariate analysis). Abbreviations: CNS, central nervous system, PD, progressive disease.
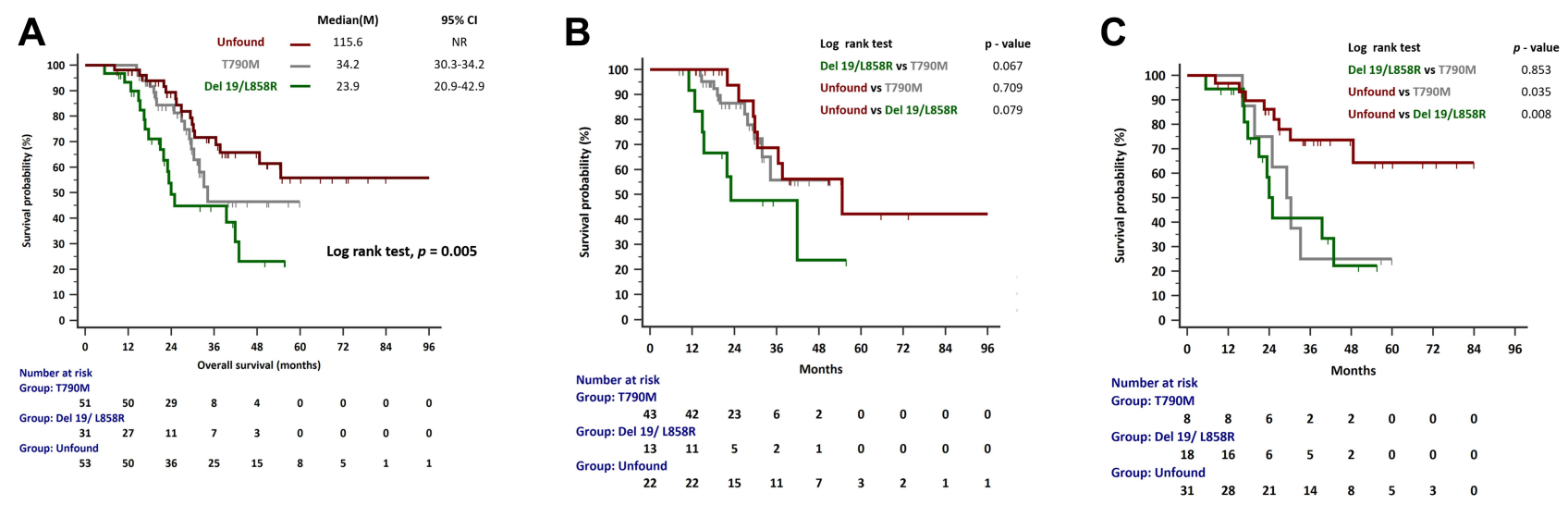

Figure 4 Kaplan-Meier curves for overall survival $(\mathbf{A})$ in the entire cohort, $(\mathbf{B})$ with osimertinib treatment, and $(\mathbf{C})$ without osimertinib treatment.

Initial liver metastasis $(p=0.017)$ and other metastasis $(p=0.023)$ were also correlated with the incidence of T790M mutation. The overall survival was the longest amongst the undetected group, followed by the shedding T790M group and then the shedding Del 19/L858R group $(p=0.005)$.

Several previous studies that focused on tissue rebiopsy have reported the likely appearance of acquired
T790M mutation in patients with EGFR-mutated NSCLC. ${ }^{13-15,20}$ Moreover, a previous meta-analysis showed that the T790M mutation was more frequent in patients with a Del 19 mutation than in patients with an L858R mutation (53\% versus $36 \%$; OR, $1.87 ; p<$ 0.001). ${ }^{12}$ A longer PFS with the first-line EGFR-TKI treatment seemed to be associated with secondary T790M prevalence; ${ }^{13,14}$ however, the AUC of PFS more 
than 11-13 months associated with EGFR T790M was only around $0.6 .^{13,14}$ Published data supporting age as a predictor of $7790 \mathrm{M}$ mutation are conflicting. Dal Maso et al reported that age younger than 65 years was significantly correlated with the acquired T790M mutation. ${ }^{19}$ In contrast, Kaburagi et al reported a positive correlation between T790 mutation and age older than 75 years. ${ }^{20}$

Tissue biopsy is more affected by tumor heterogeneity than liquid biopsy since tissue biopsy specimens originate from different sites. The majority are malignant pleural effusions or lung tissue specimens. ${ }^{15,16,20,26}$ Until now, it was uncertain whether the T790M detection rate was different at different re-biopsy sites. ${ }^{13,18,20}$ Furthermore, one study reported that as many as $19.5 \%$ of relapsed patients with NSCLC did not accept re-biopsy, and in $25.6 \%$ of rebiopsy patients, too few tumor cells were suitable for molecular analysis. ${ }^{27}$ Therefore, the $\mathrm{T} 790 \mathrm{M}$ results derived from tumor re-biopsy carry a potential risk of selection bias.

Studies have increasingly applied liquid biopsy to investigate the driver mutation in patients with NSCLC, given that liquid biopsy may overcome the issues of tumor heterogeneity, re-biopsy difficulty, and inadequacy of tumor quality. ${ }^{28,29}$ However, only limited data are available regarding the associations of patient characteristics with the detection of the T790M mutation in liquid biopsy results. In this study, we found no significant differences in T790M detection associated with age, EGFR Del 19/ L858R mutation, PFS, or immediate liquid biopsy. Wu et al also indicated that the T790M acquisition rate was no difference between patients who received and patients who did not receive chemotherapy before re-biopsy. ${ }^{30}$ Furthermore, in the present study, patients with an uncommon $E G F R$ mutation tended to be less likely to develop a T790M mutation (37.0\% for exon 19 deletion, $41.3 \%$ for L858R, and 0 for uncommon mutation); this finding was consistent with the results of another recent study. ${ }^{14,31}$

The cobas test (v2) of circulating cfDNA has been found to detect $61 \%$ of tumor specimens with the T790M mutation. ${ }^{17}$ We found that T790M mutation detection was correlated with the number of metastatic sites, occurring in up to $51.4 \%$ of patients with $\geq$ four metastatic sites. One possible explanation for this finding is that EGFR mutation adenocarcinoma patients with more metastatic sites may have a higher tumor burden and are more likely to shed more cancer cells into their bloodstream. ${ }^{17,21,22}$
The more a drug can inhibit a target, the higher the probability of resistance changing through a different pathway. Indeed, a preclinical study revealed that afatinib could partially block the growth of lung adenocarcinoma cell lines harboring the T790M mutation. ${ }^{32}$ In a series of 263 successful re-biopsies, Lee et al found that the T790M mutation was developed in $41 \%$ of patients in the afatinib group, a rate which was lower than those for the gefitinib $(55 \%)$ and erlotinib groups $(57 \%)(p=0.026) .{ }^{15}$ We also found that the $7790 \mathrm{M}$ mutation rate after afatinib therapy $(33.3 \%)$ was lower than that (40\%) after gefitinib/erlotinib, although this difference did not reach statistical significance.

In the multivariate analysis, which considered EGFR mutation type (Del 19 or L858R), age, metastatic sites, EGFR-TKI used (afatinib or gefitinib/erlotinib), PFS, and the timing of liquid biopsy, we observed a higher incidence of liver metastases in T790M-positive patients; this result echoed that of a previous re-biopsy report. ${ }^{14}$ The association of other metastasis (ie, pericardial effusion and chest wall, spleen, pancreas, kidney, peritoneum, and soft tissue metastasis) with higher rates of T790M acquired resistance is a previously unreported finding of particular interest. We also found that patients with T790M acquired resistance had specific progressive patterns of higher liver and bone metastases and higher chances of having more than two PD sites, whereas CNS progression was inversely correlated with T790M mutation. Hata et al also found a lower frequency of T790M mutation in cases of CNS progression compared to cases of thoracic lesions. The relative rarity of $\mathrm{T} 790 \mathrm{M}$ mutation in the $\mathrm{CNS}$ may be due to the low CNS drug penetration of first- and secondgeneration EGFR-TKIs. ${ }^{33}$

The anatomic location and tumor load influence the shedding of EGFR-mutated cfDNA. ${ }^{34}$ In our study population, no plasma shedding of EGFR mutations (undetected group) seemed to be a useful predictive factor for overall survival. For EGFR-mutated lung adenocarcinoma patients accepting EGFR-TKI treatment, Taus et al showed that PFS was significantly longer in patients without detectable EGFR cfDNA than in those with detectable $E G F R$ cfDNA $(295$ versus 55 days; $p<0.001){ }^{22}$ Lin et al showed that patients with tumors positive for the EGFR T790M mutation and not shedding cfDNA in the plasma had the longest PFS while taking osimertinib. ${ }^{35}$ Our research extends this application and found that for patients with advanced EGFR-mutated adenocarcinoma resistant to first-line EGFR-TKI, the overall survival was 
longest amongst the undetected group, followed by the shedding T790M group and then the shedding Del 19/ L858R group ( $p=0.005)$.

Previous studies have reported that the cobas test did not detect the T790M mutation in the plasma CfDNA of around $30 \%-40 \%$ of patients with a T790M-positive tumor test result. ${ }^{17,36}$ In the subgroup analysis with osimertinib treatment of the present study (Figure 4B), the survival curves of the undetected and shedding T790M groups were found to twist together. This might indicate that some of the undetected group patients had T790Mpositive tumors that did not shed CfDNA into their plasma. However, this cannot explain the whole picture of the undetected group patients, and the lower tumor load after first-line EGFR-TKI may have been another reason for their results. In other words, the undetected group patients either had a false-negative result regarding T790M positivity or a true negative result for T790M mutation with lower tumor load. Therefore, among the patients who did not take osimertinib, those in the undetected group had the most prolonged overall survival, and there were no differences observed in the survival of patients in the shedding Del 19/L858R and shedding T790M groups (Figure 4C).

This study had some limitations. First, the data were obtained from a single institution. Second, there are no clinical/preclinical studies that mention the mechanism of initial metastatic sites and progression patterns with secondary EGFR T790M mutation. Third, the mechanisms of acquired resistance to first-line EGFR-TKI in the undetected group were unclear because such resistance was related to molecular and pathophysiological resistance mechanisms and relied on the shedding of CfDNA. Fourth, there were only a few patients who received a tissue re-biopsy in our cohort. Therefore, we were unable to identify the true negative rate of T790M mutation. The liquid biopsies have a false-negative T790M detection rate of approximately $30 \%-40 \%{ }^{17,36}$ Similarly, a tissue biopsy is relatively invasive and still carries a risk of falsenegative results for $\mathrm{T} 790 \mathrm{M}$ due to inter-and intra-tumor heterogeneity. ${ }^{37}$ Regarding cancer heterogeneity, liquid biopsy offers an objective result regarding the T790M mutation's clinical factors.

\section{Conclusion}

Initial liver metastasis and other metastasis may be independent factors associated with secondary EGFR T790M mutation; in this study, T790M-positive lung adenocarcinoma was associated with higher rates of liver, bone, or multiple site progression. Furthermore, shedding EGFR Del19/L858R mutant cfDNA, shedding $E G F R$ T790M mutant cfDNA, and not shedding EGFR T790M mutant CfDNA are prognostic factors for patients with EGFR-mutated lung adenocarcinoma acquired resistance to first-line EGFR-TKI. In this study, the group in which EGFR T790M mutant CfDNA was undetected had the best overall survival.

\section{Abbreviations}

AUC, area under the curve; CfDNA, cell-free DNA; CI, confidence interval; CNS, central nervous system; Del 19, EGFR 19 deletion; ECOG-PS, Eastern Cooperative Oncology Group performance status; EGFR, epidermal growth factor receptor; PFS, progression-free survival; IQRs, interquartile ranges; NSCLC, non-small cell lung cancer; OR, odds ratio; PD, progressive disease; ROC, receiver operating characteristic; TKI, tyrosine kinase inhibitor.

\section{Ethics Approval and Consent to Participate}

The Investigational Review Board of China Medical University Hospital approved this study (CMUH 109REC-054), and written informed consents from patients were waived because it was a non-interventional retrospective study. We confirmed that the data of patients were maintained with confidentiality. The study was conducted in accordance with the Declaration of Helsinki.

\section{Consent for Publication}

All authors have reviewed and approved the manuscript for publication.

\section{Acknowledgments}

The authors wish to thank Chia-Ing Li for her technical help in the statistical analysis.

\section{Funding}

There is no funding to report.

\section{Disclosure}

The authors report no conflicts of interest for this work. 


\section{References}

1. Mayekar MK, Bivona TG. Current landscape of targeted therapy in lung cancer. Clin Pharmacol Ther. 2017;102(5):757-764. doi:10.1002/cpt.810

2. Mok TS, Wu Y-L, Thongprasert S, et al. Gefitinib or CarboplatinPaclitaxel in Pulmonary Adenocarcinoma. $N$ Eng $J$ Med. 2009;361 (10):947-957. doi:10.1056/NEJMoa0810699

3. Rosell R, Carcereny E, Gervais R, et al. Erlotinib versus standard chemotherapy as first-line treatment for European patients with advanced EGFR mutation-positive non-small-cell lung cancer (EURTAC): a multicentre, open-label, randomised Phase 3 trial. Lancet Oncol. 2012;13(3):239-246. doi:10.1016/S1470-2045(11)70393-X

4. Shen Y-C, Tseng G-C, Tu C-Y, et al. Comparing the effects of afatinib with gefitinib or Erlotinib in patients with advanced-stage lung adenocarcinoma harboring non-classical epidermal growth factor receptor mutations. Lung Cancer. 2017;110:56-62. doi:10.1016/j. lungcan.2017.06.007

5. Sequist LV, Yang JC-H, Yamamoto N, et al. Phase III Study of Afatinib or Cisplatin Plus Pemetrexed in Patients With Metastatic Lung Adenocarcinoma With EGFR Mutations. J clin oncol. 2013;31 (27):3327-3334. doi:10.1200/JCO.2012.44.2806

6. Sharma SV, Bell DW, Settleman J, Haber DA. Epidermal growth factor receptor mutations in lung cancer. Nat Rev Cancer. 2007;7 (3):169-181. doi:10.1038/nrc2088

7. Mok TS, Wu Y-L, Ahn M-J, et al. Osimertinib or PlatinumPemetrexed in EGFR T790M-Positive Lung Cancer. $N$ Eng $J$ Med. 2017;376(7):629-640. doi:10.1056/NEJMoa1612674

8. Soria J-C, Ohe Y, Vansteenkiste J, et al. Osimertinib in Untreated EGFR -Mutated Advanced Non-Small-Cell Lung Cancer. $N$ Eng $J$ Med. 2018;378(2):113-125. doi:10.1056/NEJMoa1713137

9. Oxnard GR, Hu Y, Mileham KF, et al. Assessment of Resistance Mechanisms and Clinical Implications in Patients With EGFR T790M-Positive Lung Cancer and Acquired Resistance to Osimertinib. JAMA oncol. 2018;4(11):1527-1534. doi:10.1001/ jamaoncol.2018.2969

10. Nakagawa K, Garon EB, Seto T, et al. Ramucirumab plus erlotinib in patients with untreated, EGFR-mutated, advanced non-small-cell lung cancer (RELAY): a randomised, double-blind, placebo-controlled, phase 3 trial. Lancet Oncol. 2019;20(12):1655-1669. doi:10.1016/ S1470-2045(19)30634-5

11. Tokaca N, Barth S, O'Brien M, et al. Molecular Adequacy of ImageGuided Rebiopsies for Molecular Retesting in Advanced Non-Small Cell Lung Cancer: A Single-Center Experience. J Thoracic Oncol. 2018;13(1):63-72. doi:10.1016/j.jtho.2017.09.1958

12. Liang H, Pan Z, Wang W, et al. The alteration of T790M between 19 del and L858R in NSCLC in the course of EGFR-TKIs therapy: a literature-based pooled analysis. J Thorac Dis. 2018;10(4):2311. doi:10.21037/jtd.2018.03.150

13. Huang Y-H, Hsu K-H, Tseng J-S, et al. The association of acquired T790M mutation with clinical characteristics after resistance to first-line epidermal growth factor receptor tyrosine kinase inhibitor in lung adenocarcinoma. Cancer Res Treat. 2018;50(4):1294. doi:10.4143/crt.2017.512

14. Lin Y-T, Chen J-S, Liao W-Y, et al. Clinical outcomes and secondary epidermal growth factor receptor (EGFR) T790M mutation among first-Line gefitinib, erlotinib and afatinib-treated non-small cell lung cancer patients with activating EGFR mutations. Int $j$ Cancer. 2019;144(11):2887-2896. doi:10.1002/ijc.32025

15. Lee K, Kim Y, Jung HA, et al. Repeat biopsy procedures and T790M rates after afatinib, gefitinib, or erlotinib therapy in patients with lung cancer. Lung Cancer. 2019;130:87-92. doi:10.1016/j.lungcan.2019. 01.012

16. Suda K, Murakami I, Sakai K, et al. Heterogeneity in resistance mechanisms causes shorter duration of epidermal growth factor receptor kinase inhibitor treatment in lung cancer. Lung Cancer. 2016;91:36-40. doi:10.1016/j.lungcan.2015.11.016
17. Jenkins S, Yang JC-H, Ramalingam SS, et al. Plasma ctDNA Analysis for Detection of the EGFR T790M Mutation in Patients with Advanced Non-Small Cell Lung Cancer. J Thoracic Oncol. 2017;12(7):1061-1070. doi:10.1016/j.jtho.2017.04.003

18. Kawamura T, Kenmotsu H, Omori S, et al. Clinical Factors Predicting Detection of T790M Mutation in Rebiopsy for EGFRMutant Non-small-cell Lung Cancer. Clin Lung Cancer. 2018;19 (2):e247-e252. doi:10.1016/j.cllc.2017.07.002

19. Dal Maso A, Lorenzi M, Roca E, et al. Clinical Features and Progression Pattern of Acquired T790M-positive Compared With T790M-negative EGFR Mutant Non-small-cell Lung Cancer: catching Tumor and Clinical Heterogeneity Over Time Through Liquid Biopsy. Clin Lung Cancer. 2020;21(1):1-14. doi:10.1016/j.cllc.2019. 07.009

20. Kaburagi T, Kiyoshima M, Nawa T, et al. Acquired EGFR T790M Mutation After Relapse Following EGFR-TKI Therapy: A Population-based Multi-institutional Study.. Anticancer Res. 2018;38(5):3145-3150. doi:10.21873/anticanres. 12577

21. Del Re M, Petrini I, Mazzoni F, et al. Incidence of T790M in Patients With NSCLC Progressed to Gefitinib, Erlotinib, and Afatinib: A Study on Circulating Cell-free DNA. Clin Lung Cancer. 2020;21 (3):232-237. doi:10.1016/j.cllc.2019.10.003

22. Taus Á, Camacho L, Rocha P, et al. Dynamics of EGFR Mutation Load in Plasma for Prediction of Treatment Response and Disease Progression in Patients With EGFR-Mutant Lung Adenocarcinoma. Clin Lung Cancer. 2018;19(5):387-394. doi:10.1016/j.cllc.2018.03. 015

23. Asamura H, Chansky K, Crowley J, et al. The International Association for the Study of Lung Cancer Lung Cancer Staging Project: proposals for the revision of the $\mathrm{N}$ descriptors in the forthcoming 8th edition of the TNM classification for lung cancer. J Thoracic Oncol. 2015;10(12):1675-1684. doi:10.1097/JTO.000000 0000000678

24. Oken MM, Creech RH, Tormey DC, et al. Toxicity and response criteria of the Eastern Cooperative Oncology Group. Am J Clin Oncol. 1982;5(6):649-656. doi:10.1097/00000421-198212000-00014

25. Thress KS, Brant R, Carr TH, et al. EGFR mutation detection in ctDNA from NSCLC patient plasma: a cross-platform comparison of leading technologies to support the clinical development of AZD9291. Lung Cancer. 2015;90(3):509-515. doi:10.1016/j.lungcan. 2015.10.004

26. Wu S-G, Liu Y-N, Tsai M-F, et al. The mechanism of acquired resistance to irreversible EGFR tyrosine kinase inhibitor-afatinib in lung adenocarcinoma patients. Oncotarget. 2016;7(11):12404. doi:10. 18632/oncotarget.7189

27. Chouaid C, Dujon C, Do P, et al. Feasibility and clinical impact of re-biopsy in advanced non small-cell lung cancer: a prospective multicenter study in a real-world setting (GFPC study 12-01). Lung Cancer. 2014;86(2):170-173. doi:10.1016/j.lungcan.2014.08.016

28. Planchard D, Popat S, Kerr K, et al. Correction to: "Metastatic nonsmall cell lung cancer: ESMO Clinical Practice Guidelines for diagnosis, treatment and follow-up". Ann Oncol. 2019;30(5):863-870. doi:10.1093/annonc/mdy474

29. Rolfo C, Mack PC, Scagliotti GV, et al. Liquid Biopsy for Advanced Non-Small Cell Lung Cancer (NSCLC): A Statement Paper from the IASLC. J Thoracic Oncol. 2018;13(9):1248-1268. doi:10.1016/ j.jtho.2018.05.030

30. Wu S-G, Chiang C-L, Liu C-Y, et al. An observational study of acquired EGFR T790M-dependent resistance to EGFR-TKI treatment in lung adenocarcinoma patients in Taiwan. Front Oncol. 2020;10:1481. doi:10.3389/fonc.2020.01481

31. John T, Akamatsu H, Delmonte A, et al. EGFR mutation analysis for prospective patient selection in AURA3 phase III trial of osimertinib versus platinum-pemetrexed in patients with EGFR T790M-positive advanced non-small-cell lung cancer. Lung Cancer. 2018;126: 133-138. doi:10.1016/j.lungcan.2018.10.027 
32. Kim Y, Ko J, Cui Z, et al. The EGFR T790M mutation in acquired resistance to an irreversible second-generation EGFR inhibitor. $\mathrm{Mol}$ Cancer Ther. 2012;11(3):784-791. doi:10.1158/1535-7163.MCT-110750

33. Hata A, Katakami N, Yoshioka H, et al. Spatiotemporal T790M Heterogeneity in Individual Patients with EGFR-Mutant Non-SmallCell Lung Cancer after Acquired Resistance to EGFR-TKI. J Thoracic Oncol. 2015;10(11):1553-1559. doi:10.1097/JTO.0000000000000647

34. Bettegowda C, Sausen M, Leary RJ, et al. Detection of Circulating Tumor DNA in Early- and Late-Stage Human Malignancies. Sci Transl Med. 2014;6(224):224ra24. doi:10.1126/scitranslmed.3007094

35. Lin -C-C, Shih J-Y, Yu C-J, et al. Outcomes in patients with non-small-cell lung cancer and acquired Thr790Met mutation treated with osimertinib: a genomic study. Lancet Respir Med. 2018;6 (2):107-116. doi:10.1016/S2213-2600(17)30480-0
36. Oxnard GR, Thress KS, Alden RS, et al. Association Between Plasma Genotyping and Outcomes of Treatment With Osimertinib (AZD9291) in Advanced Non-Small-Cell Lung Cancer. J clin oncol. 2016;34(28):3375. doi:10.1200/JCO.2016.66.7162

37. Chiang C-L, Huang H-C, Shen C-I, et al. Post-Progression Survival in Secondary EGFR T790M-Mutated Non-Small-Cell Lung Cancer Patients With and Without Osimertinib After Failure of a Previous EGFR TKI. Target Oncol. 2020;15(4):503-512. doi:10.1007/s11523020-00737-7

\section{Publish your work in this journal}

OncoTargets and Therapy is an international, peer-reviewed, open access journal focusing on the pathological basis of all cancers, potential targets for therapy and treatment protocols employed to improve the management of cancer patients. The journal also focuses on the impact of management programs and new therapeutic agents and protocols on patient perspectives such as quality of life, adherence and satisfaction. The manuscript management system is completely online and includes a very quick and fair peer-review system, which is all easy to use. Visit http://www.dovepress.com/ testimonials.php to read real quotes from published authors. 